\title{
Analysis of prognostic factors in 171 patients with myxofibrosarcoma of the trunk and extremities: a cohort study
}

\author{
Huanmei Liu, Xinxin Zhang, Shuguang Zhang, Shengji Yu \\ National Cancer Center/National Clinical Research Center for Cancer/Cancer Hospital, Chinese Academy of Medical Sciences and Peking Union \\ Medical College, Beijing, China \\ Contributions: (I) Conception and design: H Liu; (II) Administrative support: S Yu; (III) Provision of study materials or patients: X Zhang; (IV) \\ Collection and assembly of data: H Liu, S Zhang; (V) Data analysis and interpretation: H Liu; (VI) Manuscript writing: All authors; (VII) Final \\ approval of manuscript: All authors. \\ Correspondence to: Shengji Yu. National Cancer Center/National Clinical Research Center for Cancer/Cancer Hospital, Chinese Academy of Medical \\ Sciences and Peking Union Medical College, 17 Panjiayuan Nanli, Chaoyang District, Beijing 100021, China. Email: shengjiyu@126.com.
}

Background: Myxofibrosarcoma (MFS) of the trunk and extremities has unique clinical features. However, it is not clear which indicators are the influencing factors of recurrence, metastasis, and survival of trunk and limb MFS. The aim of the present study was to analyze clinical features and prognosis of trunk and limb MFS.

Methods: The data of 171 patients with MFS of the trunk and extremities and a median follow-up period of 67 months from January 1999 to July 2018 were retrospectively analyzed. Risk factors for survival, recurrence and metastasis following resection of MFS of trunk and extremities were analyzed. The KaplanMeier method (log-rank test) was used for the univariate analysis and a Cox regression model was used for the multivariate analysis.

Results: The median age of the patients was 53 years; there were 111 males and 60 females. A total of 132 cases had French Federation of Cancer Centers grade 1, 24 cases had grade 2, and 15 cases had grade 3 MFS. The 3-year recurrence, 3-year metastasis, and 5-year survival rates were $29.2 \%, 19.3 \%$, and $93.6 \%$, respectively. Kaplan-Meier survival analysis showed that the surgical margin $\left(\chi^{2}=22.228, \mathrm{P}<0.001\right)$ and tumor size $\left(\chi^{2}=6.697, \mathrm{P}=0.010\right)$ were associated with recurrence. The surgical margin $\left(\chi^{2}=12.353, \mathrm{P}<0.001\right)$ and CD44 expression $\left(\chi^{2}=5.227, \mathrm{P}=0.022\right)$ were associated with metastasis. The multivariate analysis showed that the surgical margin [hazard ratio $(\mathrm{HR})=3.635,95 \%$ confidence interval $(\mathrm{CI}): 1.883-7.016, \mathrm{P}<0.001$ ] and tumor size ( $\mathrm{HR}=1.889,95 \% \mathrm{CI}$ : $1.039-3.435, \mathrm{P}=0.037)$ were risk factors for local recurrence. In addition, the surgical margin ( $\mathrm{HR}=4.475,95 \% \mathrm{CI}: 1.918-10.438, \mathrm{P}=0.001)$ and presence of $\mathrm{CD} 44(\mathrm{HR}=3.406,95 \%$ CI: $1.462-8.405, \mathrm{P}=0.005$ ) were risk factors for distant metastasis.

Conclusions: A negative surgical margin can be reduced effectively the rate of recurrence and metastasis in patients with MFS of the trunk and limbs. In addition, CD44 may be used to assess the metastatic risk of patients with MFS.

Keywords: Myxofibrosarcoma (MFS); trunk; extremities; recurrence-free survival; metastasis-free survival; prognostic factors

Submitted Jun 17, 2021. Accepted for publication Aug 12, 2021.

doi: $10.21037 / \mathrm{atm}-21-3587$

View this article at: https://dx.doi.org/10.21037/atm-21-3587 


\section{Introduction}

Myxofibrosarcoma (MFS) is one of the most aggressive sarcomas. MFS was first described in 1977 was it was included in the World Health Organization (WHO) classification as a distinct pathological entity (1). Histologically, MFS is usually composed of spindle cells and polygonal cells with nuclear atypia. Pathologically, there are many similar morphologic neoplasms, such as low-grade fibromyxoid sarcoma, myxoid dermatofibrosarcoma, ossified fibromyxoid neoplasms, and superficial angiomyxoma (2). Therefore, MFS can be easily misdiagnosed as these tumors, which makes the diagnosis of MFS difficult. MFS usually occurs in the extremities and limbs, and is characterized by slow, painless growth of the mass. Despite aggressive surgical treatment, the rate of postoperative recurrence is still high, and the overall 5 -year survival rate is only about $60-70 \%$ (3).

The factors influencing the recurrence, metastasis and survival of MFS have attracted attention. Dewan et al. retrospectively analyzed the clinical data of 174 patients with MFS from UK, and they think highlighting the unpredictable nature of MFS (4). Gily et al. retrospectively analyzed the clinical data of 109 patients with MFS from Austria, and their results support that patients with high-grade MFS had a poorer prognosis with respect to disease specific survival/ distant metastasis free survival than low-grade MFS (5). Sanfilippo et al. retrospectively analyzed the clinical data of 158 patients with MFS from Italy, The results suggested that the factors related to recurrence and metastasis include tumor size, grade and surgical margins (6). In conclusion, there are fewer factors for assessing disease progression in MFS.

In the present study, we evaluated the clinical data of 171 patients with MFS of the trunk and extremities to analyze the prognostic factors associated with recurrence, metastasis, and survival. Our research has the following characteristics: (I) the object of study is from China; (II) the tumor occurred in the trunk and extremities; (III) tumor biomarkers (CD44, Ki67, CD68) were added to the model. The aim of the present study was to analyze prognosis of trunk and limb MFS.

We present the following article in accordance with the STROBE reporting checklist (available at https://dx.doi. org/10.21037/atm-21-3587).

\section{Methods}

\section{Patients}

All procedures performed in this study involving human participants were in accordance with the Declaration of Helsinki (as revised in 2013). This study was approved by the Ethics Committee of National Cancer Center/National Clinical Research Center for Cancer/Cancer Hospital, Chinese Academy of Medical Sciences and Peking Union Medical College (No.: NCC20200-/341). Because of the retrospective nature of the research, the requirement for informed consent was waived. A total of 254 patients with MFS of the trunk and limbs were registered in the Cancer Hospital of the Chinese Academy of Medical Sciences from March 1999 to July 2018. All patients' pathology report could be re-evaluated, and pathologically confirmed as MFS according to the 2013 WHO Classification of Soft Tissue and bone tumors (1). Infantile fibrosarcoma, lowgrade malignant fibromyxoid sarcoma, sclerosing epithelial fibrosarcoma, and protuberant dermatofibrosarcoma were excluded. Among them, 32 cases were complicated with other tumors, 24 cases had metastasis, 7 cases had no operation, and 20 cases had incomplete clinical data. A total of 171 patients were included in the present study. A total of 151 cases underwent operation in our hospital, and 20 cases received pathological consultations in our hospital. A total of 161 MFS cases were confirmed for the first time by operation in our hospital.

The observation indexes included age, sex, primary tumor site, tumor size, French Federation of Cancer Centers (FNCLCC) grade, tumor biomarkers (CD44, Ki67, CD68), and adjuvant therapy. The primary sites of the tumors were recorded according to the trunk and limbs. Imaging data were obtained from our hospital or other hospitals. Margin status was determined by two specialized soft-tissue pathologists (BL, SS). Margins were classified as $\mathrm{R} 0$ when the tumor was at least $1 \mathrm{~mm}$ away from the resection margins, otherwise margin status was classified as R1. If gross residual tumor was present at the margin, it was classified as R2. The tumor tissues were fixed with $10 \%$ formaldehyde solution for $24 \mathrm{~h}$, dehydrated, and embedded in paraffin. The expression of CD44, Ki67, and CD68 was detected by immunohistochemistry. Adjuvant therapy included radiotherapy and chemotherapy. Tumorfree survival time was defined as the time from the operative day to recurrence, metastasis, death, or the last follow up. Recurrence-free survival time and metastasis-free survival time were defined as the time from the operative day to recurrence, metastasis, death, or the last follow up. Overall survival time referred to the time from the operative day to the patient's death from cancer or the last follow up. The surgical margin was negative for $\mathrm{R} 0$ resection based on the 
Union for International Cancer Control classification; that is, there was no tumor residual under the microscope. The last follow up was until December 31, 2020. Overall survival was defined as from the time of the diagnosis of MFS to death or the last follow up.

\section{Adjuvant chemoradiotherapy regimen}

Radiotherapy was given to the patients with a large tumor volume, positive surgical margin, or postoperative local recurrence. Intraoperative radiotherapy was 10-22 Gy. The conventional dose of postoperative radiotherapy was 50-60 Gy, which was completed in 25-30 courses. Chemotherapy was administered after metastasis or multiple relapses, including $\mathrm{AI}$ regimen (epirubicin + ifosfamide), $\mathrm{AD}$ regimen (epirubicin + dacarbazine), and MAID regimen (mesna + adriamycin + ifosfamide + dacarbazine) .

The patients or their family was contacted by telephone, WeChat, QQ (Tencent's Internet-based instant messaging software), or email for follow up. The start point of follow up was the operative day, and the end point was the last follow-up date or the date of patient death.

\section{Statistical analysis}

All data were collated and analyzed by SPSS 22.0 statistical software (IBM, Armonk, NY, USA). General data were analyzed by frequency distribution. The age of onset was compared using a non-parametric test. The Kaplan-Meier method (log-rank test) was used for the univariate analysis. Factors assessed included age, sex, primary tumor site, tumor size, FNCLCC grade, CD44, Ki67, CD68, and adjuvant therapy.

A Cox regression model was used for the multivariate analysis. Multivariate Cox regression analysis was used where more than one predictive factor reached significance in the univariate analysis. The test level was set at $\alpha=0.05$ for the bilateral tests.

\section{Results}

\section{Demographic and tumor characteristics of the study population}

There were 111 males and 60 females aged from 16 to 80 years in the cohort, with a median age of 53 years. There were 88 cases of MFS in the trunk, including 1 case in the supraclavicular region, 8 cases in the shoulder, 2 cases in the shoulder and back, 13 cases in the chest, 16 cases in the abdomen, 9 cases in the back, 10 cases in the chest and back, 2 cases in the waist, 2 cases in the sacrum, 1 case in the lumbosacral region, 2 cases in the waist and back, 3 cases in the hip, 3 cases in the iliac fossa, 6 cases in the hip, and 5 cases in the groin. There were 83 MFS cases in the limbs, including 9 cases in the upper arm, 4 cases in the elbow, 10 cases in the forearm, 44 cases in the thigh, 6 cases in the knee, and 15 cases in the calf. The maximum diameters of the tumors ranged from 1.2 to $18 \mathrm{~cm}$. In 89 cases, the largest diameter was less than $5 \mathrm{~cm}$, and in 82 cases, the largest diameter was greater than $5 \mathrm{~cm}$. There were 132 cases of FNCLCC grade 1, 24 cases of grade 2, and 15 cases of grade 3 MFS.

Magnetic resonance imaging showed that the lesions were mainly isointense-hypointense on the T1-weighted images (T1WIs), had mixed intensity on the $\mathrm{T} 2$-weighted images (T2WIs), and obvious gyri-like high signals could be seen. Striped low-signal segregation was observed on the T1WIs and T2WIs. The boundaries of the lesions were clear, and bone invasions could be partially seen.

Microscopically, spindle-shaped cells were observed to have a uniform size and shape, low cell mass, and unclear membrane boundaries, and they were separated by collagen fibers arranged in a parallel pattern. Scattered giant tumor cells and inflammatory cell infiltration were noted, and mitotic figures were easily observed. The number of mitotic figures ranged from 5/10 to 46/10 high power field (HPF), and some cases were accompanied by massive necrosis, interstitial sclerosis, and vitreous degeneration. Well-differentiated tumor spindle cells showed a uniform appearance without deep-stained nuclei. The cells were curved or cross-bundled to form a typical "herringbone" pattern. Differentiated MFS was characterized by dense, disordered, small-sized, and round- or oval-shaped cells, and a small amount of collagen fibers; mitosis was common. Tumor biomarkers were positive for CD 44 protein in 22 cases, Ki67 >20\% in 56 cases, and CD68 in 36 cases.

\section{Survival analysis}

The number of cases in the Chinese Academy of Medical Sciences during the study period determined the sample size. All 171 patients underwent extensive MFS resection. Large lesions were repaired with flaps or free skin grafting. Two patients underwent amputation. The surgical margin was positive in 20 cases, and 50 cases developed recurrence. The 3 -year recurrence rate was $29.2 \%$, and 33 cases had 


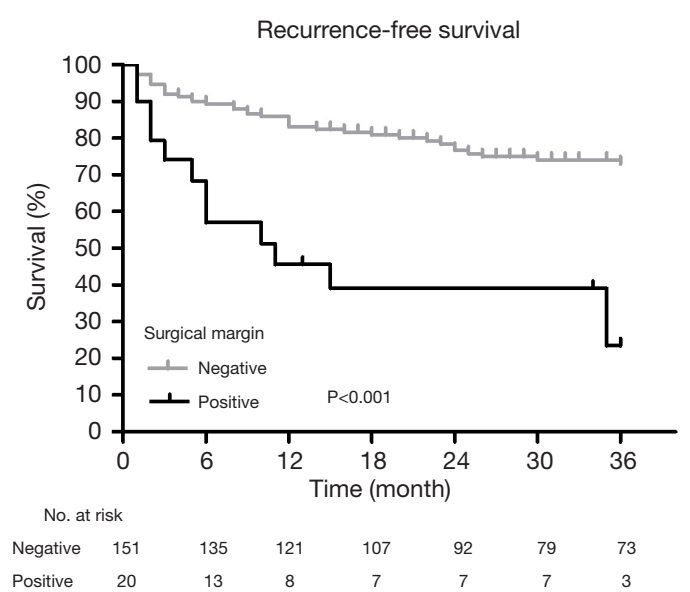

Figure 1 Recurrence-free survival analysis of patients based on the surgical margin using the Kaplan-Meier method.

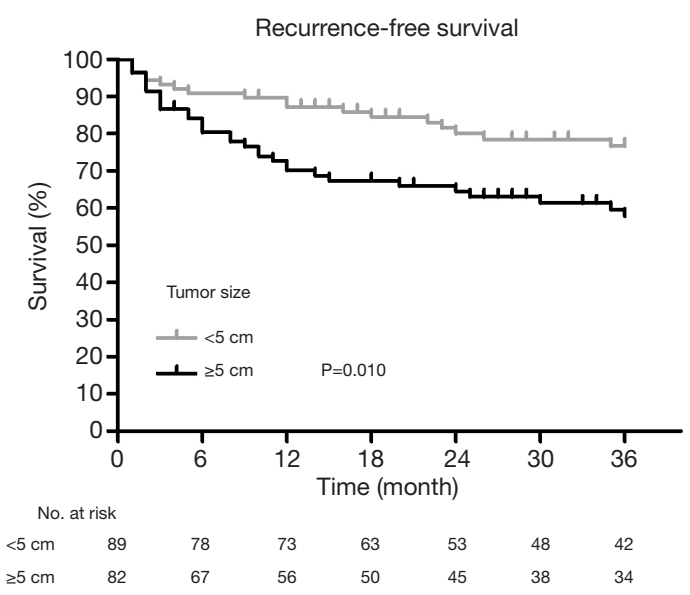

Figure 2 Recurrence-free survival analysis of patients based on the tumor size using the Kaplan-Meier method.

metastasis. There were 26 cases of pulmonary metastasis, of which 3 cases involved multiple metastases (lung and local lymph node metastasis in 1 case; lung, thorax, abdomen, and right subphrenic metastasis in 1 case; and lung, pleura, and abdominal metastasis in 1 case). The remaining 23 cases involved only pulmonary metastasis. Bone metastasis occurred in the sacrum, femur, and lumbar spine in 3 cases, respectively. Abdominal metastasis occurred in 1 case and back metastasis occurred in 1 case. The followup period ranged from 12 to 142 months, with a median follow-up period of 67 months. Sixty cases were treated with adjuvant therapy ( 35 cases with radiotherapy alone, 12 cases with chemotherapy alone, 13 cases with radiotherapy plus chemotherapy). Among the 21 patients treated with

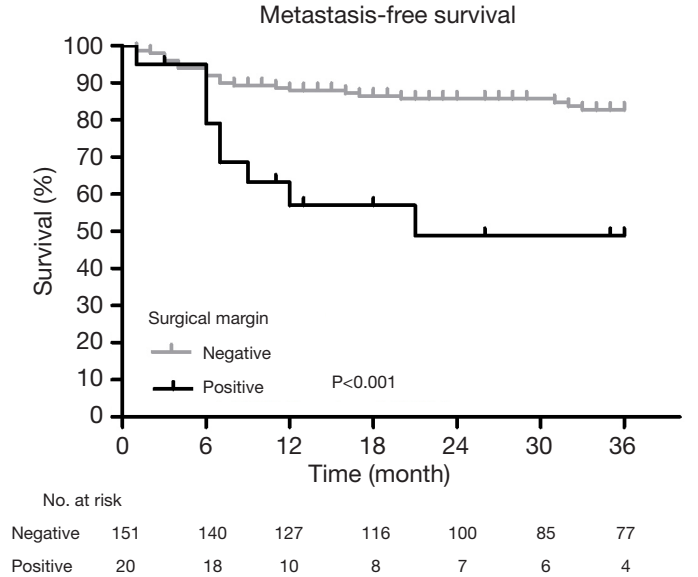

Figure 3 Metastasis-free survival analysis of patients based on the surgical margin using the Kaplan-Meier method.

adjuvant therapy in the first operation, 9 cases had no recurrence or metastasis, and 52 patients were treated with adjuvant therapy after postoperative recurrence or metastasis. The 3-year recurrence rate of the 171 patients was $29.2 \%$, the 3 -year metastasis rate was $19.3 \%$, and the 5 -year survival rate was $93.6 \%$.

The Kaplan-Meier survival analysis showed that the surgical margin $\left(\chi^{2}=22.228, \mathrm{P}<0.001\right)$ and tumor size $\left(\chi^{2}=6.697, \mathrm{P}=0.010\right)$ were associated with recurrence (Figures 1,2). The surgical margin $\left(\chi^{2}=12.353, \mathrm{P}<0.001\right)$ and CD44 expression $\left(\chi^{2}=5.227, \mathrm{P}=0.022\right)$ were associated with metastasis (Figures 3,4). There were no factors associated with 5 -year survival in the included characteristics. These modalities are shown in Table 1. Multivariate analysis of prognostic factors for recurrence included surgical margin and size. Multivariate analysis of prognostic factors for metastasis included surgical margin and CD44. The multivariate analysis showed that the surgical margin [hazard ratio $(\mathrm{HR})=3.635, \mathrm{P}<0.001]$ and tumor size $(\mathrm{HR}=1.889$, $\mathrm{P}=0.037$ ) were risk factors for local recurrence, as shown in Table 2. In addition, the surgical margin ( $\mathrm{HR}=4.475$, $\mathrm{P}=0.001)$ and presence of $\mathrm{CD} 44(\mathrm{HR}=3.406, \mathrm{P}=0.005)$ were risk factors for distant metastasis of MFS in the trunk and extremities, as shown in Table 3.

\section{Discussion}

\section{Recurrence, metastasis, and survival}

With the improvement of surgical standardized treatment, the recurrence rate of MFS had decreased. In their study, 


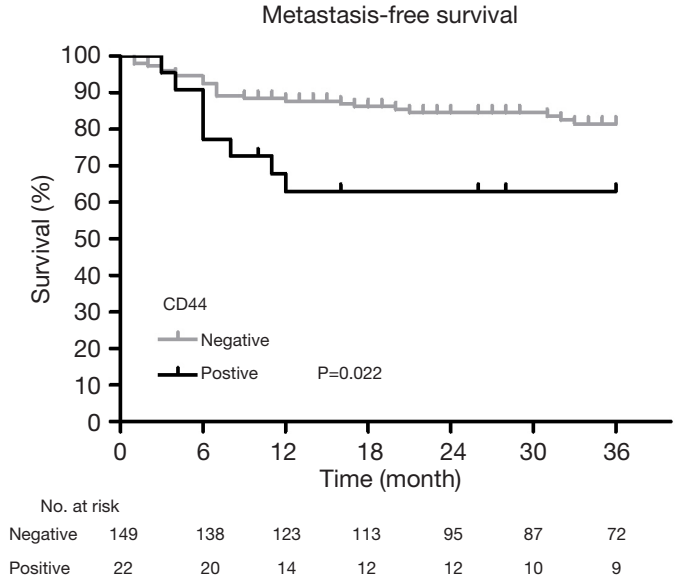

Figure 4 Metastasis-free survival analysis of patients based on the CD44 using the Kaplan-Meier method.

Yoshimoto et al. reported that the local recurrence rate in 162 patients was $34.4 \%$ (7), Gilg et al. reported that the 3 -year recurrence rate of 109 patients in their study was $10 \%$ (5), and Scoccianti et al. reported that the 5-year recurrence rate of 75 patients with MFS in their study was $23.2 \%$ (8). The results showed that there were 50 cases of recurrent MFS in 171 patients, with a 3-year recurrence rate of $29.2 \%$. Among them, the 3 -year recurrence rate was $65 \%$ in 20 patients with positive margins and $24.5 \%$ in 151 patients with negative margins, which was generally consistent with existing reports $(5,7,8)$.

The metastatic rates of MFS reported by different studies vary greatly. Yoshimoto et al. reported that the distant metastasis rate of MFS was $12.2 \%$ in their study (7), but Gilg et al. reported that the 3-year survival rate of 162 MFS patients was $23 \%$ in their study (5). Moreover, in their study, Mühlhofer et al. reported that the metastasis rate of MFS was $36.2 \%$, and the mean metastasis time was 20.4 months (9). In their study Sanfilippo et al. reported that the MFS metastasis rate was $65 \%$ (6). In the present study, the metastasis rate was $19.3 \%$, and pulmonary metastasis occurred in 26 cases. In addition, there were 3 cases of bone metastasis in the sacrum, femur, and lumbar vertebrae, respectively, in this group. Tumors were located in the lumbosacral, thigh, and lumbar back. It was found that tumors associated with bone metastasis were adjacent to the affected bone. One case presented with lymph node metastasis in the inguinal region and lung metastasis.

The survival rates of patients with MFS reported in several studies were relatively similar. Gilg et al. reported a
3 -year survival rate of $79 \%$ and a 5 -year survival rate of $76 \%$ in 162 patients with MFS (5). The 5-year survival rate of 158 MFS patients reported by Sanfilippo et al. was 77\% (6), while the 5-year survival rate of 172 MFS patients reported by Dewan et al. was 63\% (4). However, our study found that the 5 -year survival rate of the 171 patients was $93.6 \%$, which was much higher than these reports. The Kaplan-Meier survival analysis showed that adjuvant therapy improved the survival rate of the patients. However, the multivariate analysis did not support any independent factors influencing the 5-year survival rate. This may be related to the increased survival rate of patients and decreased number of deaths. In clinical practice, doctors often administer adjuvant therapy to patients with a poor prognosis.

\section{Surgical margin and tumor size}

Surgery is the preferred treatment for MFS. The methods for extensive resection of MFS are similar to the surgical methods for soft-tissue sarcoma, including a $3-5-\mathrm{cm}$ resection of the normal tissue adjacent the tumor (10). When the skin is involved, a $3-\mathrm{cm}$ area adjacent to the affected skin is excised (11). For patients whose tumors invade nerves and blood vessels, if the important nerves and blood vessels can be dissected and separated during the operation, the limbs can be preserved by internal and external irradiation. Amputation was performed in patients with an advanced tumor grade and tumor rupture in general. In the case of the chest wall, it is often necessary to remove the ribs, repair the chest wall, and use a free musculocutaneous flap for repair. After excision of the large abdominal wall, the defect can be repaired by a skin flap or medical patch (12).

Multiple studies have confirmed that surgical margin and tumor size are closely related to the prognosis of patients with MFS. In their study, Dewan et al. showed a correlation between tumor size and MFS survival (4). Fujiwara et al. reported that 52 of their cases had resection margins $\geq 10 \mathrm{~mm}, 195$ cases had resection margin of 0 $9.9 \mathrm{~mm}$, and 31 cases had negative resection margins in 191 cases of undifferentiated pleomorphic sarcoma and 87 cases of MFS (13). The results showed that margins were associated with progression-free survival, but the clinical data of MFS were not analyzed separately in this study (13), so it is not possible to directly infer whether margin is statistically related to MFS. Sanfilippo et al. found that tumor size and margins were statistically significant predictors of survival (6). Gilg et al. showed that R1 status 
Table 1 Demographic and tumor characteristics of the 171 cases of myxofibrosarcoma of the trunk and extremities

\begin{tabular}{|c|c|c|c|c|c|c|c|c|c|c|}
\hline Factors & Case No. & $\begin{array}{c}\text { 3-year recurrence } \\
\text { rate }(\%)\end{array}$ & $\chi^{2}$ & $P$ value & $\begin{array}{c}\text { 3-year metastasis } \\
\text { rate }(\%)\end{array}$ & $\chi^{2}$ & $P$ value & $\begin{array}{c}\text { 5-year survival } \\
\text { rate (\%) }\end{array}$ & $\chi^{2}$ & $P$ value \\
\hline \multicolumn{11}{|l|}{ Sex } \\
\hline Female & 60 & 25.0 & 0.684 & 0.408 & 19.8 & 0.02 & 0.887 & 96.7 & 1.450 & 0.229 \\
\hline Male & 111 & 21.5 & & & 18.3 & & & 91.9 & & \\
\hline \multicolumn{11}{|c|}{ Age at diagnosis (years) } \\
\hline$\geq 53$ & 88 & 30.7 & & & 18.2 & & & 94.3 & & \\
\hline \multicolumn{11}{|l|}{ Site } \\
\hline Extremity & 93 & 23.7 & 0.454 & 0.5 & 22.9 & 0.885 & 0.347 & 94.3 & 0.001 & 0.971 \\
\hline$>5$ & 82 & 39.0 & & & 24.4 & & & 91.5 & & \\
\hline \multicolumn{11}{|c|}{ FNCLCC grade } \\
\hline Grade 1 & 132 & 28.8 & 1.641 & 0.440 & 19.7 & 1.198 & 0.549 & 94.7 & 2.754 & 0.252 \\
\hline Grade 2 & 24 & 25.0 & & & 12.5 & & & 87.5 & & \\
\hline Grade 3 & 15 & 40.0 & & & 26.7 & & & 93.3 & & \\
\hline \multicolumn{11}{|l|}{ Ki67 (\%) } \\
\hline$<20$ & 115 & 29.6 & 0.142 & 0.706 & 17.4 & 1.062 & 0.303 & 94.8 & 2.031 & 0.154 \\
\hline Negative & 135 & 30.4 & 0.384 & 0.535 & 19.3 & 0.00 & 0.986 & 91.9 & 2.989 & 0.089 \\
\hline Positive & 36 & 25.0 & & & 19.4 & & & 100 & & \\
\hline \multicolumn{11}{|c|}{ Surgical margin } \\
\hline Negative & 151 & 24.5 & 22.228 & 0.001 & 25.9 & 12.353 & 0.001 & 94.0 & 1.817 & 0.178 \\
\hline Positive & 20 & 65.0 & & & 45.0 & & & 90.0 & & \\
\hline \multicolumn{11}{|c|}{ Adjuvant chemotherapy/radiation (\%) } \\
\hline No & 111 & NA & NA & NA & NA & NA & NA & 91.9 & 1.880 & 0.170 \\
\hline Yes & 60 & NA & & & NA & & & 96.7 & & \\
\hline
\end{tabular}

FNCLCC, French Federation of Cancer Centers; NA, no available statistics. 
Table 2 Multivariate analysis of prognostic factors affecting recurrence in the 171 cases of myxofibrosarcoma of the trunk and extremities

\begin{tabular}{lcc}
\hline Characteristics & $\begin{array}{c}\text { Hazard ratio } \\
(95 \% \text { confidence interval })\end{array}$ & P value \\
\hline Surgical margin & Reference & $<0.001$ \\
Negative & $3.635(1.883-7.016)$ & \\
Positive & Reference & 0.037 \\
Size $(\mathrm{cm})$ & $1.889(1.039-3.435)$ & \\
$\leq 5$ &
\end{tabular}

Table 3 Multivariate analysis of prognostic factors affecting metastasis in the 171 cases of myxofibrosarcoma of the trunk and extremities

\begin{tabular}{lcc}
\hline Characteristics & $\begin{array}{c}\text { Hazard ratio } \\
\text { (95\% confidence interval) }\end{array}$ & P value \\
\hline Surgical margin & Reference & $<0.001$ \\
Negative & $4.475(1.918-10.438)$ & \\
Positive & Reference & 0.005 \\
CD44 & 3.506 (1.462-8.405) & \\
Negative & \\
Positive &
\end{tabular}

was an independent factor in recurrence-free survival of MFS (odds ratio: 8.5, 95\% CI: 1.59-49.79) (5).

In the present study, the 20 patients with positive incision margins had a 3 -year recurrence rate of $65 \%$, which was higher than the $24.5 \%$ recurrence rate in the negative incision margin group, and a 3-year metastasis rate of $45 \%$, which was higher than the $25.9 \%$ metastasis rate in the negative incision margin group. The multivariate analysis showed that the surgical margin was an independent risk factor for recurrence and metastasis within 3 years. The results of this study showed that the tumor size was an independent factor influencing the 3 -year recurrence rate. The larger the tumor, the more extensive its invasion to surrounding tissues will be. Therefore, $\mathrm{R} 0$ resection is difficult to achieve. Therefore, the larger the tumor, the higher the recurrence rate.

\section{Immunobistochemical analysis}

Immunohistochemistry of MFS was usually positive for vimentin; partially positive for Ki67, CD44, and CD68; and negative for S-100, AE/AE3, and $\alpha$-SMA $(14,15)$. Immunohistochemical biomarkers provide an important observational index for the diagnosis and prognosis of tumors. Increasing attention has been paid to the factors related to metastasis. Ki67 is a protein encoded by the Ki67 gene and is closely associated with cell proliferation. Some studies have found that it can predict the prognosis of breast cancer patients with bone metastasis (16). However, the value of Ki67 as an indicator of the therapeutic response to adjuvant treatment in patients with soft-tissue sarcomas has been questioned (17).

CD44, a member of the cell-adhesion molecule family, is a I transmembrane glycoprotein encoded by a single gene, which is widely expressed on the surface of a variety of cells and tissues (18). CD44 can be modified to form a variety of isomers, which can be combined with a variety of ligands on the cell surface to participate in various physiological and pathological processes. Current studies have confirmed that CD44 is abnormally highly expressed in breast cancer, gastric cancer, and colorectal cancer, and is involved in the growth and aggressive behavior of these tumors (19-22). Tsuchie et al. reported (22) an average CD44-positive cell rate of $34.6 \% \pm 20.4 \%$ in 44 patients with MFS, and high CD44 expression did not increase the rate of distant metastasis in MFS patients, but shortened eventfree survival (OR: 1.032, 95\% CI: 1.010-1.053). Moreover, the recurrence-free survival of high CD44 expression was shorter than the recurrence-free survival of normally expressed CD44 in MFS patients with negative incitement margins (OR: 1.059, 95\% CI: 1.014-1.106) (22).

CD68 is a biomarker of tumor-associated macrophages (TAMs) (23). The expression of TAMs is associated with epithelial-to-mesenchymal transition (24). Under chronic inflammation, TAMs can differentiate into cells that can support tumor growth, promote extracellular matrix remodeling, angiogenesis, and immunosuppression, and enhance the invasion ability of cancer cells by secreting a variety of factors. The CD68 protein can be detected in the pathological tissue of some MFS patients, and it is distributed in the tumor nest, tumor stroma, or peri-tumor stroma.

In this study, there were 56 MFS patients (32.7\%) with Ki67 expression $\geq 20 \%$, and 36 MFS patients (21.1\%) 
were CD68 positive. There was no significant correlation between Ki67 expression (>20\%) and the recurrence or metastasis of MFS.

\section{Evaluation research}

Some studies have shown that grade is the influencing factor of MFS. Gilg et al. showed that grade was an independent factor in progression-free survival, as well as in metastasisfree survival (5). Scoccianti et al. found that higher grades were associated with higher recurrence rates (8). Factor analysis results in our study showed that there was no statistical significance between FNCLCC grade and the recurrence, metastasis, and survival of patients with MFS, which was different from Gilg's and Scoccianti's study. This is probably due to sample bias.

CD109, a TGF-b co-receptor, is reported to be preferentially expressed during the early stages of tumorigenesis in several carcinoma types (25). Some studies have reported that CD109 expression in MFS is associated with poor prognosis. CD109 was not analyzed in the present study. However, the inclusion of CD109 may change the current multivariate analysis model.

Factors influencing survival can only be calculated with a sufficient sample size. However, all tumor-related deaths in the present study were due to metastasis. In addition, a more reliable analysis model can be established for the analysis of factors related to the survival of patients after metastasis with the sample size is $\geq 50$. Therefore, no statistically significant factors affecting the survival of patients with MFS were found in the present study.

\section{Conclusions}

A positive surgical margin is an important cause of recurrence and metastasis in patients with MFS of the trunk and extremities. Extended resection is the first-choice therapy for patients with MFS. For those with a positive incision margin, appropriate adjuvant therapy may reduce the mortality rate caused by the recurrence and metastasis of tumors.

\section{Acknowledgments}

Funding: this work was supported by Capital Characterized Clinical Application Research Fund of Beijing Municipal Science and Technology Commission of China
(Z171100001017210), Beijing Hope Run Special Fund of Cancer Foundation of China (LC2016L01), and CAMS Innovation Fund for Medical Sciences (CIFMS) (2017I2M-1-005).

\section{Footnote}

Reporting Checklist: The authors have completed the STROBE reporting checklist. Available at https://dx.doi. org/10.21037/atm-21-3587

Data Sharing Statement: Available at https://dx.doi. org/10.21037/atm-21-3587

Conflicts of Interest: All authors have completed the ICMJE uniform disclosure form (available at https://dx.doi. org/10.21037/atm-21-3587). The authors report that this work was supported by Capital Characterized Clinical Application Research Fund of Beijing Municipal Science and Technology Commission of China (Z171100001017210), Beijing Hope Run Special Fund of Cancer Foundation of China (LC2016L01), and CAMS Innovation Fund for Medical Sciences (CIFMS) (2017-I2M-1-005). The authors have no other conflicts of interest to declare.

Ethical Statement: The authors are accountable for all aspects of the work in ensuring that questions related to the accuracy or integrity of any part of the work are appropriately investigated and resolved. All procedures performed in this study involving human participants were in accordance with the Declaration of Helsinki (as revised in 2013). This study was approved by the Ethics Committee of National Cancer Center/National Clinical Research Center for Cancer/Cancer Hospital, Chinese Academy of Medical Sciences and Peking Union Medical College (No.: NCC20200-/341). Because of the retrospective nature of the research, the requirement for informed consent was waived.

Open Access Statement: This is an Open Access article distributed in accordance with the Creative Commons Attribution-NonCommercial-NoDerivs 4.0 International License (CC BY-NC-ND 4.0), which permits the noncommercial replication and distribution of the article with the strict proviso that no changes or edits are made and the original work is properly cited (including links to both the formal publication through the relevant DOI and the 
license). See: https://creativecommons.org/licenses/by-nc$\mathrm{nd} / 4.0 /$.

\section{References}

1. Coindre JM. New WHO classification of tumours of soft tissue and bone. Ann Pathol 2012;32:S115-6.

2. Ghazala CG, Agni NR, Ragbir M, et al. Myxofibrosarcoma of the extremity and trunk: a multidisciplinary approach leads to good local rates of LOCAL control. Bone Joint J 2016;98-B:1682-8.

3. Oda Y, Takahira T, Kawaguchi K, et al. Low-grade fibromyxoid sarcoma versus low-grade myxofibrosarcoma in the extremities and trunk. A comparison of clinicopathological and immunohistochemical features. Histopathology 2004;45:29-38.

4. Dewan V, Darbyshire A, Sumathi V, et al. Prognostic and survival factors in myxofibrosarcomas. Sarcoma 2012;2012:830879.

5. Gilg MM, Sunitsch S, Leitner L, et al. Tumor-associated mortality and prognostic factors in myxofibrosarcoma - A retrospective review of 109 patients. Orthop Traumatol Surg Res 2020;106:1059-65.

6. Sanfilippo R, Miceli R, Grosso F, et al. Myxofibrosarcoma: prognostic factors and survival in a series of patients treated at a single institution. Ann Surg Oncol 2011;18:720-5.

7. Yoshimoto M, Yamada Y, Ishihara S, et al. Comparative Study of Myxofibrosarcoma With Undifferentiated Pleomorphic Sarcoma: Histopathologic and Clinicopathologic Review. Am J Surg Pathol 2020;44:87-97.

8. Scoccianti G, Ranucci V, Frenos F, et al. Soft tissue myxofibrosarcoma: A clinico-pathological analysis of a series of 75 patients with emphasis on the epithelioid variant. J Surg Oncol 2016;114:50-5.

9. Mühlhofer HML, Lenze U, Gersing A, et al. Prognostic Factors and Outcomes for Patients With Myxofibrosarcoma: A 13-Year Retrospective Evaluation. Anticancer Res 2019;39:2985-92.

10. Kawaguchi N, Ahmed AR, Matsumoto S, et al. The concept of curative margin in surgery for bone and soft tissue sarcoma. Clin Orthop Relat Res 2004;(419):165-72.

11. Odei B, Rwigema JC, Eilber FR, et al. Predictors of Local Recurrence in Patients With Myxofibrosarcoma. Am J Clin Oncol 2018;41:827-31.

12. Wakely PE Jr. Cytopathology of myxofibrosarcoma: a study of 66 cases and literature review. J Am Soc Cytopathol 2021;10:300-9.

13. Fujiwara T, Sumathi V, Parry M, et al. The role of surgical margin quality in myxofibrosarcoma and undifferentiated pleomorphic sarcoma. Eur J Surg Oncol 2021;47:1756-62.

14. Yoshimoto M, Yamada Y, Ishihara S, et al. Retroperitoneal Myxofibrosarcoma: A Controversial Entity. Pathol Res Pract 2020;216:152969.

15. de Gooyer JM, Versleijen-Jonkers YMH, HillebrandtRoeffen MHS, et al. Immunohistochemical selection of biomarkers for tumor-targeted image-guided surgery of myxofibrosarcoma. Sci Rep 2020;10:2915.

16. Mansouri H, Mnango LF, Magorosa EP, et al. Ki-67, p53 and BCL-2 Expressions and their Association with Clinical Histopathology of Breast Cancer among Women in Tanzania. Sci Rep 2019;9:9918.

17. Benz MR, Czernin J, Allen-Auerbach MS, et al. 3'-deoxy3'-18Ffluorothymidine positron emission tomography for response assessment in soft tissue sarcoma: a pilot study to correlate imaging findings with tissue thymidine kinase 1 and Ki-67 activity and histopathologic response. Cancer 2012;118:3135-44.

18. Isacke CM, Yarwood H. The hyaluronan receptor, CD44. Int J Biochem Cell Biol 2002;34:718-21.

19. Wittig BM, Johansson B, Zöller M, et al. Abrogation of experimental colitis correlates with increased apoptosis in mice deficient for CD44 variant exon 7 (CD44v7). J Exp Med 2000;191:2053-64.

20. Shipitsin M, Campbell LL, Argani P, et al. Molecular definition of breast tumor heterogeneity. Cancer Cell 2007;11:259-73.

21. Matuschek C, Lehnhardt M, Gerber PA, et al. Increased CD44s and decreased CD44v6 RNA expression are associated with better survival in myxofibrosarcoma patients: a pilot study. Eur J Med Res 2014;19:6.

22. Tsuchie H, Emori M, Miyakoshi N, et al. Prognostic Impact of CD44 Expression in Patients With Myxofibrosarcoma. In Vivo 2019;33:2095-102.

23. Tanaka R, Umeyama Y, Hagiwara H, et al. Keloid patients have higher peripheral blood endothelial progenitor cell counts and CD34+ cells with normal vasculogenic and angiogenic function that overexpress vascular endothelial growth factor and interleukin-8. Int J Dermatol 2019;58:1398-405.

24. Solinas G, Germano G, Mantovani A, et al. Tumorassociated macrophages (TAM) as major players of the cancer-related inflammation. J Leukoc Biol 
Page 10 of 10

2009;86:1065-73.

25. Emori M, Tsukahara T, Murata K, et al. Prognostic impact of CD109 expression in myxofibrosarcoma. J Surg Oncol

Cite this article as: Liu H, Zhang X, Zhang S, Yu S. Analysis of prognostic factors in 171 patients with myxofibrosarcoma of the trunk and extremities: a cohort study. Ann Transl Med 2021;9(16):1322. doi: 10.21037/atm-21-3587
Liu et al. Myxofibrosarcoma of the trunk and extremities

2015;111:975-9.

(English Language Editor: R. Scott) 